\title{
Eficacia de las técnicas de control de la impulsividad en la prevención de la adicción a videojuegos
}

\section{Effectiveness of impulsivity control techniques to videogame addiction prevention}

\author{
Clara Marco \\ Mariano Chóliz \\ Departamento de Psicología Básica. Facultad de Psicología \\ Universidad de Valencia. España.
}

Rec (26 de febrero de 2016) Acept (16 febrero de 2017)

\begin{abstract}
Resumen
El objetivo principal de esta investigación consistió en el análisis de la eficacia de las técnicas de control de la impulsividad para la prevención de la adicción a videojuegos añadidas a un programa de prevención previamente validado (PrevTec 3.1). Los resultados se tomaron en cuatro momentos: línea base, pre-test (primera sesión), post-test (última sesión) y seguimiento (a los tres meses). Los resultados indican que, mientras los grupos que permanecen en lista de espera no presentan cambios ni en el patrón de uso ni en la dependencia de videojuegos, aquellos en los que se aplica el programa sí que experimentan un descenso significativo. Las dos modalidades del programa de prevención son eficaces en la reducción de las variables analizadas, pero los resultados se mantienen más consistentes durante el seguimiento a quienes se entrenaron las técnicas de control de la impulsividad.

Palabras clave: adicción a videojuegos, adicciones tecnológicas, prevención, técnicas de control de la impulsividad.
\end{abstract}

\begin{abstract}
The main objective of this research has been the analysis of the effectiveness of some impulsivity control techniques to prevent videogame addiction. It has compared the results obtained with a prevention program that it had been already validated with the same program in which additional impulsiveness control techniques were added. Four periods were selected for the analysis: baseline, pre-test, post-test and follow-up. Two experimental conditions were designed: a) conventional prevention program, and b) program with impulsiveness control techniques. Results were compared with a group control (waiting list). Results indicate that, while groups that remain on waiting list do not present changes in pattern of use, those which the program was applied decrease significantly. The two modalities of prevention program were effective in the reduction of the analyzed variables, but the changes produced by the implementation of impulsive control techniques were more consistent and lasting in time.
\end{abstract}

Key words: videogame addiction, technological addictions, prevention, impulsivity control techniques.

\footnotetext{
Correspondencia: Mariano Chóliz Facultad de Psicología, Universidad de Valencia. Avda Blasco Ibáñez, 21 46010-Valencia, España.Email: Mariano.Choliz@uv.es

Agradecimientos: Los resultados presentados en este artículo han sido obtenidos gracias a un proyecto de investigación financiado por la Fundación Universitaria Oriol-Urquijo.
} 


\section{Introducción}

Las tecnologías como Internet, el móvil y los videojuegos representan instrumentos muy útiles en nuestra vida cotidiana, sin embargo, también pueden tener sus inconvenientes. Su propio diseño es susceptible de afectar a la capacidad de control, lo cual, junto con otros factores personales y ambientales, facilita su uso abusivo o inadecuado, pudiendo llegar a generar situaciones problemáticas y producirse una conducta adictiva (Saldaña, 2001), especialmente entre los menores. En este sentido, las adicciones tecnológicas (Griffiths, 1995) son el tipo más reciente de adicciones comportamentales. El elemento sustancial que caracteriza a todas ellas y que comparten con las drogodependencias es precisamente la dependencia, un patrón de conducta en el que se evidencia una pérdida de control y que en el caso de las tecnologías se establece como un consumo excesivo $\mathrm{y}$ disfuncional.

En este artículo nos hemos centrado en los videojuegos, que en la sociedad actual constituyen una importante forma de entretenimiento, liderando el segmento de ocio digital e interactivo en España (Asociación Española de Distribuidores y Editores de Software de Entretenimiento ADESE, 2011), especialmente entre los adolescentes y los jóvenes, quienes suelen dedicar una parte importante de su tiempo a jugar con ellos.

Sin poner en duda la importancia del juego para el desarrollo personal, en el aprendizaje o como una actividad con un destacado papel motivacional, el abuso del mismo también puede acarrear problemas. Algunos videojuegos inducen a un patrón de juego caracterizado por un consumo excesivo, que en algunas personas llega a interferir con pautas de conducta higiénicas o saludables y hasta perturbar las relaciones familiares en algunos casos (Patim, 2009). Es preciso hacer constar que, en gran medida, el patrón de consumo excesivo está favorecido por las propias características que tienen muchos videojuegos y que son, precisamente, algunas de sus principales virtudes -sensación de dominio y autodeterminación, estética agradable y fantástica, feedback de actuación, compromiso con otras personas (en el caso de los multijugador), absorción de la realidad, etcétera-. Tanto estas propiedades motivacionales, como las consecuencias derivadas del uso excesivo, pueden llegar a provocar una imperiosa necesidad de jugar en algunas personas.

En la actualidad se considera que existe suficiente evidencia empírica, clínica y científica (Griffiths, 2000) de que el juego en exceso constituye una actividad que puede convertirse en problemática (Tejeiro y Bersabé, 2002) y en algunos casos adictiva (Griffiths, 2008a), especialmente en juegos online, en los que se trata de una actividad expansiva y sin fin, en la que las posibilidades de acción son innumerables (Chappell, Eatough, Davies y Griffiths, 2006). En esta dirección, diversos estudios han confirmado la existencia de un subgrupo de usuarios de videojuegos que cumple los criterios de dependencia de los mismos (Chiu, Lee y Huang, 2004; Gentile, 2009; Griffiths y Hunt, 1998; Grüsser, Thalemann y Griffiths, 2007; Lemmens, 2006; Lemmens, Valkenburg y Pet, 2009; Meerkerk, Van Den Eijnden, Vermulst y Garretsen, 2009; Peukert, Sieslack, Barth, y Batra, 2010; Van den Eijnden, Van Rooij y Meerkerk, 2007; Van Rooij, Schoenmakers, van de Eijnden, y van de Mheen, 2010; Van Rooij y Van den Eijnden, 2007). Y aunque el problema del uso adictivo de los videojuegos no es un fenómeno nuevo (Keepers, 1990), la introducción del componente online en la actual generación de juegos probablemente ha aumentado el alcance del problema. Y es que también debe tenerse en cuenta el tipo o género de videojuego, ya que las diferencias entre unos y otros tipos de juegos tienen implicaciones en su potencial adictivo (Elliot, Golub, Ream y Dunlap, 2012). De hecho, diversos estudios han demostrado que los videojuegos MMORPG se asocian con grados superiores de dependencia (Castronova, 2005; Griffiths, Davies y Chappell, 2004; Lee et al., 2007; Smyth, 2007; Van Cleave, 2010).

La persona adicta a los videojuegos presenta problemas que se extienden a todas las parcelas de su vida -salud, familia, escuela y relaciones sociales- (Echeburua y Requesens, 2012). Y es que una de las consecuencias más evidentes de este problema es la alteración de un estilo de vida socialmente adaptativo, perturbando la organización temporal diaria (Van Rooij, 2011), dedicando una cantidad de tiempo excesiva al juego, o provocando severas interferencias con otras actividades (Bainbridge, 2007; Egli y Meyers, 1984; Griffiths y Hunt, 1998). Pueden reducirse las relaciones interpersonales (Allison, von Wahlde, Shockley y Gabbard, 2006) - de hecho, algunos jugadores prefieren las relaciones online que las de la vida real (Peters y Maleski, 2008)- y producirse problemas y discusiones en las relaciones familiares y de pareja, puede darse una disminución del rendimiento académico o laboral (Chiu, Lee y Huang, 2004; Griffiths y Hunt, 1998; Phillips, Rolls, Rouse y Griffiths, 1995; Rehbein, Kleimann y Mößle, 2010; Skoric, Teo y Neo, 2009), o el abandono de otras actividades de ocio (Batthyány, Müller, Benker y Wölfling, 2009; Griffiths et al., 2004; King y Delfabbro, 2009; Peters y Malesky, 2008; Rehbein et al., 2010; Yee, 2006). Otras de las consecuencias negativas del abuso y dependencia de los videojuegos consisten en la alteración del patrón de sueño (Batthyány et al., 2009), 
deficiencias nutricionales y falta de higiene, y en algunos casos extremos, comportamientos suicidas (Rehbein et al., 2010) y homicidas (Hart et al., 2009; Tanner, 2007). De forma similar a otros trastornos adictivos, la adicción a los videojuegos presenta componentes de craving, alteraciones del humor, síntomas de abstinencia (ansiedad, ira, irritación y depresión), problemas con el control de los impulsos, y un aumento del uso a pesar de las consecuencias negativas y las pérdidas en otros ámbitos de la vida (Grüsser et al., 2007).

\section{Adolescencia, impulsividad y fascinación por la tecnología}

La adolescencia representa un periodo crítico en lo que se refiere a las conductas de riesgo en general y, concretamente, al uso de sustancias y al riesgo de desarrollar otras conductas adictivas (Arnett, 1999; Steinberg, 2005). Se trata también de una de las etapas más relevantes en el desarrollo de la personalidad y, muy especialmente, del desarrollo cerebral. Y es que hasta la tercera década de la vida continúan desarrollándose zonas cerebrales como la corteza prefrontal -responsable de la función ejecutiva y de la autorregulación de la conducta (Rubia, 2004; Spear, 2000a, 2000b; Weinberger, Elvevag y Giedd, 2005)-.

Por otro lado, no podemos olvidar que los adolescentes son muy sensibles al momento y al entorno social en el que viven, en el que estas herramientas se han convertido en un elemento imprescindible en sus vidas (Castellana, SánchezCarbonell, Beranuy y Graner, 2006; Machargo, Luján, León, López y Martín, 2003), y que las TIC (móvil, videojuegos e Internet) cuentan con una serie de características que resultan especialmente atractivas para ellos, cumpliendo una serie de funciones particularmente relevantes en esta etapa del desarrollo, como pueden ser la búsqueda de identidad y autonomía, la presencia del grupo de iguales, el incremento de las relaciones interpersonales o la desinhibición.

\section{Prevención de las Adicciones Tecnológicas}

El problema con el que nos encontramos en la actualidad es que, a pesar de que existe una demanda social y de las familias de las personas afectadas para abordar el problema del uso abusivo y la dependencia de los videojuegos (y otras tecnologías), todavía no se ha producido una conciencia clara en el ámbito sociosanitario de que se trate de un problema sobre el que se deban tomar los recursos terapéuticos y preventivos apropiados de forma similar a los de cualquier otro trastorno adictivo. Mientras tanto, la tecnología se va desarrollando, haciéndose cada vez más disponible, accesible, amable, atractiva y funcional, y la industria presiona por que se utilice de forma excesiva. En este contexto, hay personas (generalmente jóvenes y adolescentes) que se ven prisioneros de unas herramientas que, en lugar de mejorar la calidad de vida, se la están deteriorando.

La adicción a los videojuegos representa un problema real para algunas personas $\mathrm{y}$, aunque los afectados son una minoría, representan a muchos adolescentes. A pesar de esto, no existen programas específicos para la prevención de la dependencia de los videojuegos. Y sin duda, el uso de las nuevas tecnologías por los adolescentes y jóvenes confirma la necesidad de dotar a estos y a los adultos de conocimientos básicos que les ayuden a educar y acompañar a los menores en su uso (Jiménez et al., 2012).

PrevTec 3.1 (Chóliz, 2011) es un programa de prevención de las adicciones tecnológicas (móvil, Internet y videojuegos) cuya eficacia se ha demostrado en diversos estudios (Marco, Chóliz y Martínez, 2012; Marco, Villanueva y Chóliz, 2010; Villanueva, Marco y Chóliz, 2010a, 2010b, 2012). Dicho programa trabaja la prevención de la dependencia de videojuegos, que se consigue estableciendo un patrón de juego apropiado. En principio no se pretende la abstinencia absoluta, puesto que la actividad no es perjudicial en sí misma y con el desarrollo de algunas estrategias adecuadas es factible lograr un uso controlado de los videojuegos. Para lograr el patrón de uso adecuado, algunas de las indicaciones más relevantes que deberían tenerse en cuenta son las siguientes (Chóliz, Marco y Chóliz, 2014): 1. Establecer un horario de juego. 2. Complementariamente, deberán establecerse qué actividades se llevarán a cabo en los momentos en los que no se va a jugar. 3. Evitar los juegos online, al menos hasta que se tenga una organización del tiempo libre que impida dedicar mucho tiempo a dichos videojuegos. 4. No instalar la consola ni el ordenador en la habitación. 5. Los padres deben conocer los videojuegos.

Esta investigación pretende llevar a cabo un estudio controlado que tenga como objetivo principal el análisis de la eficacia de varias técnicas de control de la impulsividad en el programa de Prevención de Adicciones Tecnológicas PrevTec 3.1 (Chóliz, 2011). En concreto, se pretende comparar la eficacia diferencial del módulo de videojuegos del programa PrevTec 3.1 tradicional, frente al mismo programa con técnicas de control de la impulsividad adicionales, y frente al grupo control (lista de espera). 


\section{Método}

\section{Participantes:}

La muestra final la constituyen 1110 adolescentes de entre 9 y 16 años, siendo la edad media 12,37 años ( $\mathrm{Sx}=$ 1.875). Todos los participantes están escolarizados en centros educativos públicos o privados de la ciudad de Valencia y su área metropolitana. En concreto, el 38,3\% son estudiantes de Educación Primaria y el 61,7\% de Educación Secundaria Obligatoria. De los trece centros que participaron, nueve son centros públicos y cuatro son privados.

\section{Instrumentos}

Para realizar la medida antes de la implementación del programa de prevención, evaluar el impacto del mismo y el efecto tras varios meses desde su aplicación, se administraron diversos cuestionarios: cuestionario pretest, cuestionario en la primera sesión del programa, retest en la tercera sesión $\mathrm{y}$ cuestionario de seguimiento. Se midieron las siguientes variables:

a) Datos sociodemográficos y escolares: edad, sexo, nombre del colegio o instituto y curso.

b) Parámetros del uso de videojuegos: frecuencia de juego semanal, tiempo de juego diario entre semana y en fin de semana y juegos más usados.

c) Parámetros del uso de videojuegos online: frecuencia y tiempo de juego online entre semana y en fin de semana, tiempo dedicado a los videojuegos online en comparación con el resto de juegos, número de conexiones diarias para consultar la evolución del juego online, y juegos más usados.

d) Percepción subjetiva de dependencia de los videojuegos: se preguntó mediante una escala que oscila de 0 a 100 , cuál es su grado dependencia de los videojuegos.

e) Dependencia de los videojuegos: se evaluó la dependencia de los videojuegos mediante el Test de Dependencia de Videojuegos (TDV) (Chóliz y Marco, 2011). El TDV es un instrumento diagnóstico de la dependencia de los videojuegos, basado en los principales criterios diagnósticos del trastorno por dependencia de sustancias del Manual Diagnóstico y Estadístico de los Trastornos Mentales en su cuarta versión revisada (DSM-IV-TR) de la American Psychiatric Association (APA). Consta de 25 ítems y presenta una consistencia interna elevada (alfa de Cronbach $=.94)$. Los primeros 14 ítems se responden mediante una escala tipo Likert que oscila entre 0 y 4, referida a la frecuencia ( 0 "nunca"; 1 "rara vez"; 2 "a veces"; 3 "con frecuencia" y 4 "casi siempre"), y en los 11 ítems restantes se pregunta por el grado de acuerdo o desacuerdo, a través de una escala tipo Likert que oscila entre 0 y 4 (0 "totalmente en desacuerdo"; 1 "un poco en desacuerdo"; 2 "neutral"; 3 "un poco de acuerdo" y 4 "totalmente de acuerdo"), sobre un conjunto de afirmaciones relacionadas con los videojuegos. El cuestionario está compuesto por cuatro factores: Abstinencia (malestar que se presenta cuando el sujeto no puede jugar con videojuegos y uso de los mismos para aliviar problemas psicológicos); Abuso y Tolerancia (jugar progresivamente más que al principio y de forma excesiva); Problemas ocasionados por los videojuegos (consecuencias negativas del uso excesivo de los videojuegos); y Dificultad en el control (dificultades para dejar de jugar, a pesar de que no sea funcional ni adecuado hacerlo en ese momento o situación).

\section{Diseño y procedimiento}

En este trabajo se ha utilizado un diseño mixto o de medidas parcialmente repetidas, que combina el diseño de medidas repetidas y el diseño entre-sujetos. Existe una variable de medidas repetidas (evaluación inicial o pre-test inicial, pre-test, post-test y seguimiento) con dos condiciones o grupos experimentales (programa tradicional y programa con técnicas de control de la impulsividad) y un grupo control (en lista de espera).

Los participantes, por grupos, fueron asignados aleatoriamente a cada una de las condiciones experimentales: 1. Programa de Prevención en la condición tradicional (PrevTec 3.1). En esta condición los grupos realizaban las sesiones del programa con las actividades "tradicionales", a saber, horario semanal de uso de videojuegos procurando no jugar todos los días ni hacerlo fuera del tiempo programado, jugar después de realizar otras obligaciones, realizar actividades alternativas en el tiempo libre, enseñar los videojuegos preferidos a los padres, y evitar los videojuegos multijugador online, y 2. Programa de Prevención con técnicas de control del impulso adicionales. En esta condición, además de las actividades ya comentadas, se pedía a los alumnos que pusieran en práctica técnicas de control de la impulsividad. Dichas técnicas fueron las siguientes: a) Antes de ponerse a jugar, tienen que esperar entre cinco y diez minutos. Pueden aprovechar ese tiempo para valorar si es un buen momento para jugar, si tienen otras cosas que hacer o si estaba programado ese tiempo para el juego. b) Antes de comenzar con el juego tienen que ponerse una 
alarma (en el móvil, reloj, despertador, etc.) que suene un poco antes de que termine el tiempo programado para jugar, y que sirva como señal para parar de jugar. 3. Grupo control-lista de espera. En esta tercera condición, los grupos realizaban una evaluación inicial (pre-test), y unos meses después se realizaban las sesiones del programa, bien en la condición tradicional bien con las técnicas de control de impulsividad adicionales.

El $44.7 \%$ de la muestra $(n=494)$ realizó el programa tradicional y el $55.3 \%(n=612)$ realizó el programa de prevención con técnicas de control de impulsividad adicionales. El grupo que realiza el programa tradicional tiene una media de edad de 12.64 años $\left(\mathrm{S}_{\mathrm{x}}=1.61\right)$, el $50.7 \%$ son chicos y el $49.3 \%$ chicas. En el grupo con técnicas adicionales, la media de edad es de 12.15 años $\left(S_{x}=2.04\right)$, el $46.3 \%$ son chicos y el $53.7 \%$ son chicas.

El procedimiento que se siguió para reclutar la muestra consistió en contactar mediante un correo electrónico, con los diferentes colegios de la ciudad de Valencia y su área metropolitana en que había alumnos cursando desde $5^{\circ}$ de Primaria hasta $4^{\circ}$ de la ESO. Para ello, se accedió al listado del Registro Estatal de Centros Docentes no Universitarios y se invitó a participar a alrededor de doscientos centros, de los cuales participaron trece. La selección de la muestra, por tanto, se realizó mediante un muestro no probabilístico, sin norma o circunstancial.

De los trece centros que participaron, siete constituyeron el grupo experimental en un primer momento, cinco de ellos la lista de espera, y en un centro se repartieron los grupos entre las condiciones experimentales y control -lista de espera-. En los centros que configuraron la lista de espera, se realizó un pretest y se acordaron las fechas para realizar el programa a partir del mes de enero de 2012. En el resto de los centros se comenzó la aplicación del programa directamente, sin realizar el pretest, a partir del mes de noviembre de 2011.

La asignación de los centros como grupo experimental y grupo en lista de espera, así como las condiciones del grupo experimental (programa con técnicas de control de la impulsividad adicionales o sin ellas) se realizó de forma aleatoria.

La implementación del programa se llevó a cabo entre noviembre de 2011 y junio de 2012, por parte de psicólogos y estudiantes de último curso de Psicología previamente entrenados para ello. La aplicación del programa se realizó en presencia del tutor responsable, en el aula y en horario de tutorías, con intención de no interferir en el desarrollo de las clases, y estas se realizaban en los mismos grupos de clase. Cada sesión tuvo una duración aproximada de unos cincuenta minutos, y las sesiones del programa estuvieron separadas entre sí por una semana.

En el caso de los centros con pretest, entre el pretest y la primera sesión del programa pasaron entre tres y cuatro meses. Desde la última sesión del programa hasta el seguimiento pasaron entre dos y cinco meses.

El proyecto fue aprobado por la comisión ética de la Facultad de Psicología de la Universidad de Valencia

\section{Análisis de los datos}

Todos los datos se analizaron mediante el paquete estadístico SPSS para Windows (versión 19.0).

En este artículo se presentan los resultados hallados en diversas pruebas t y análisis de varianza (ANOVA) y de covarianza (ANCOVA) de medidas repetidas para el contraste de medias, ANOVA y pruebas Post Hoc ((Student-NewmanKeuls (S-N-K) y Diferencia Mínima Significativa (DMS)).

\section{Resultados}

\section{Diferencias entre grupos antes de la aplicación del programa de prevención}

Con el fin de comprobar la homogeneidad de los dos grupos de participantes (condición tradicional versus programa con técnicas adicionales), se realizaron pruebas " $t$ " para muestras independientes y pruebas $\mathrm{c}^{2}$.

Respecto a las variables sociodemográficas, no existen diferencias significativas entre los grupos en la variable sexo $\left(\mathrm{c}^{2}=1.315 ;\right.$ g.l. $\left.=1 ; \mathrm{p}=.251\right)$, pero sí en la edad $\left(t_{(1107,793)}=4.473\right.$; $\mathrm{p}=.000 ; \mathrm{d}=0.27)$.

En cuanto a las variables relacionadas con el patrón de uso de los videojuegos, los resultados de las pruebas $t$ indican que no existen diferencias entre los dos grupos en el patrón de uso de los videojuegos, es decir, ni en la frecuencia de uso ni en el tiempo dedicado a ellos -tanto entre semana como en fin de semana-. Aparecen diferencias estadísticamente significativas en el grado de dependencia percibida y en la puntuación total del TDV, que habrá que tener en cuenta y corregir para hacer los dos grupos comparables entre sí.

\section{Análisis de la eficacia de las dos modalidades del programa de prevención de la adicción a videojuegos}

Evolución del grupo en lista de espera 
El grupo en lista de espera está formado por 471 alumnos de entre 9 y 16 años, siendo la edad media 12.33 años $\left(\mathrm{S}_{\mathrm{x}}=1.927\right)$.

Los resultados de las pruebas $t$ de diferencias entre medias (Tabla 1) indican que entre el pretest y el test, realizado entre tres y cuatro meses después del pretest (sin realizar entre las dos medidas ningún tipo de intervención), no aparecen diferencias estadísticamente significativas en ninguna de las variables estudiadas.

Tabla 1. Evolución del patrón de uso y dependencia de los videojuegos del grupo en lista de espera (pretest-test)

\begin{tabular}{lcc}
\hline & Pretest & Test \\
\hline Frecuencia de juego & $2.54(2.48)$ & $2.69(2.46)$ \\
Tiempo de juego LJ & $67.73(50.19)$ & $70.28(49.32)$ \\
Tiempo de juego FS & $98.45(64.01)$ & $101.49(64.01)$ \\
Dependencia percibida & $34.80(27.52)$ & $36.68(27.89)$ \\
TDV & $26.14(20.10)$ & $27.91(20.96)$ \\
\hline
\end{tabular}

Eficacia diferencial de las dos modalidades del programa de prevención de la adicción a videojuegos

Con el fin de comparar la eficacia diferencial de las dos modalidades de intervención aplicadas, se llevaron a cabo Análisis de Varianza (ANOVAS) de medidas repetidas para cada una de las variables dependientes medidas (frecuencia de juego, tiempo de juego entre semana, tiempo de juego en fin de semana, grado de dependencia percibida, puntuación en el TDV), utilizando la corrección de Greenhouse-Geisser. Se analizaron las diferencias entre los tres momentos de medida (test-retest-seguimiento), excepto en el caso de la puntuación en el TDV, cuya medida se realizó únicamente en el test y en el seguimiento (test-seguimiento).

Puesto que se hallaron diferencias en la media de edad entre los dos grupos antes de la intervención, se realizaron dichos análisis empleando, además, un análisis de covarianza, siendo la edad la variable covariada. De este modo, se pudo controlar el efecto de las diferencias en la edad sobre las diferencias encontradas entre los grupos tras la aplicación del programa.

Frecuencia de juego

Los resultados (Tabla 2) muestran un descenso de la frecuencia de juego entre el test, el retest y el seguimiento, $y$ que dicho descenso es mayor en el grupo del programa con técnicas adicionales. Así pues, mientras que en el test y en el retest no existen diferencias estadísticamente significativas entre ambos grupos, en el seguimiento sí que existen ( $t=$ 3.705; $p=.000 ; d=0.23$ ), siendo la media de la frecuencia de juego menor en el grupo con técnicas adicionales que en el grupo del programa tradicional.

Tabla 2. Frecuencia de juego (dias por semana). Medias y desviaciones típicas

\begin{tabular}{lccc}
\hline & Test & Retest & Seguimiento \\
\hline Programa tradicional & $2.48(2.28)$ & $2.16(2.04)$ & $2.01(2.08)$ \\
$\begin{array}{l}\text { Programa con técni- } \\
\text { cas adicionales }\end{array}$ & $2.37(2.38)$ & $1.98(2.01)$ & $1.56(1.82)$ \\
\hline
\end{tabular}

Respecto al ANCOVA de medidas repetidas, la variable inter-grupo es la modalidad del programa de prevención, y la variable intra-grupo es el momento de medida. Como se puede observar en la Tabla 3, se encuentra un efecto significativo de la interacción entre ambos factores $\left(\mathrm{F}_{(1,929)}=3.470 ; \mathrm{p}=.033\right)$, así como un efecto significativo en el factor grupo $\left(\mathrm{F}_{(1)}=4.862 ; \mathrm{p}=.028\right)$ en la frecuencia de juego. El efecto del factor momento no resultó estadísticamente significativo.

Para examinar la interacción, se analizaron los efectos simples -es decir, se compararon entre sí los niveles del factor momento dentro de cada nivel del factor grupo-, usando el ajuste de los niveles críticos mediante la corrección de Bonferroni para controlar la tasa de error Tipo I (Maxwell y Delaney, 1990). Las comparaciones por pares (Tabla 4) indican que mientras en el programa tradicional no existen diferencias estadísticamente significativas entre el retest y el seguimiento, en el grupo que recibe el programa con técnicas de control de la impulsividad adicionales la frecuencia de juego continúa descendiendo entre el retest y el seguimiento. La magnitud del cambio total entre el test y el seguimiento es superior en el grupo con técnicas adicionales.

La representación gráfica de los resultados de cada uno de los apartados puede consultarse en el Anexo I.

Tiempo de juego entre semana

Se aplicó un ANCOVA de medidas repetidas (3x2) para las mediciones realizadas del tiempo de juego entre semana, con un factor entre-grupos (condición programa tradicional vs. programa con técnicas de autocontrol adicionales) y un factor momento (test-retest-seguimiento). 
Tabla 3. Frecuencia de Juego. Resultados del ANCOVA MR

\begin{tabular}{ccccccccccccc}
\hline Momento & \multicolumn{1}{c}{} & \multicolumn{4}{c}{$\begin{array}{c}\text { Interacción } \\
\text { Momento x Grupo }\end{array}$} \\
\hline $\mathrm{F}$ & $\mathrm{p}$ & $\mathrm{h}^{2}$ & $1-\mathrm{b}$ & $\mathrm{F}$ & $\mathrm{p}$ & $\mathrm{h}^{2}$ & $1-\mathrm{b}$ & $\mathrm{F}$ & $\mathrm{p}$ & $\mathrm{h}^{2}$ & $1-\mathrm{b}$ \\
0.066 & .932 & .000 & .060 & 3.470 & .033 & .033 & .641 & 4.862 & .028 & .006 & .596 \\
\hline
\end{tabular}

Tabla 4. Comparaciones por pares para la interacción Tipo de Programa (Grupo) x Momento (Variable: frecuencia de juego)

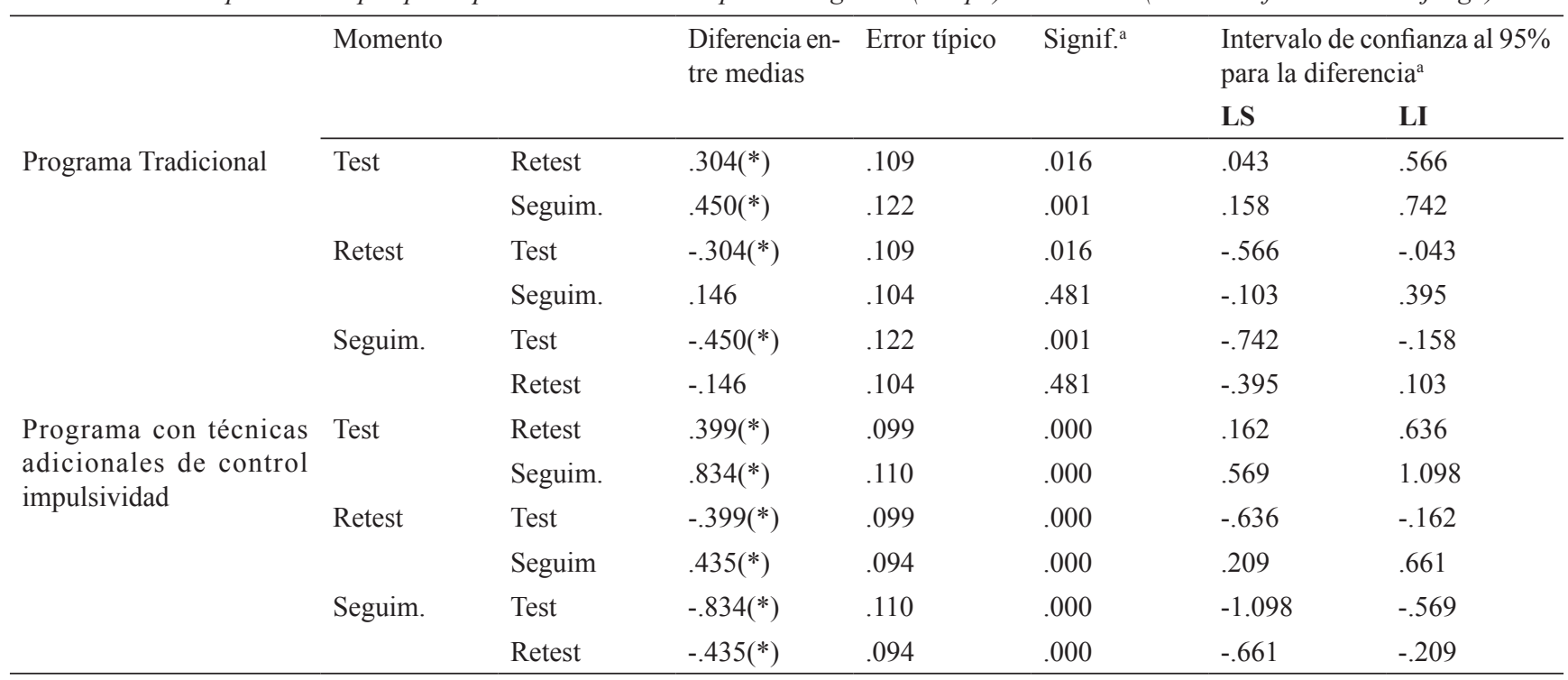

Nota. Basadas en las medias marginales estimadas.

* La diferencia de las medias es significativa al nivel .05.

a Ajuste para comparaciones múltiples: Bonferroni.

Tabla 5. Tiempo de juego entre semana (minutos). Medias y desviaciones típicas

\begin{tabular}{lccc}
\hline & Test & Retest & Seguimiento \\
\hline $\begin{array}{l}\text { Programa } \\
\text { tradicional }\end{array}$ & $68.13(49.78)$ & $36.91(52.18)$ & $34.11(51.57)$ \\
$\begin{array}{l}\text { Programa } \\
\text { con técnicas } \\
\text { adicionales }\end{array}$ & $60.29(44.47)$ & $28.92(45.54)$ & $22.14(37.62)$ \\
\hline
\end{tabular}

Los resultados (Tabla 5) muestran un descenso del tiempo de juego entre semana a lo largo de los distintos momentos de medida (test-retest-seguimiento) para los dos grupos. Puede apreciarse que el cambio es mayor en el grupo que realiza el programa con técnicas de control de la impulsividad adicionales, así mientras que en el test no existen diferencias entre grupos en esta variable, en el retest $(t=2.760 ; \mathrm{p}=.006 ; \mathrm{d}=0.17)$ y en el seguimiento $(t=4.091$; $\mathrm{p}=.000 ; \mathrm{d}=0.27)$ las diferencias son estadísticamente menores en el segundo grupo.

Los resultados del ANCOVA de medidas repetidas (Tabla 6) muestran que no existe un efecto significativo de la interacción entre ambos factores, por lo que se examinan los efectos principales. Se encuentra un efecto significativo en el factor momento $\left(\mathrm{F}_{(1.994)}=6.415 ; \mathrm{p}=.002\right)$, así como un efecto del factor grupo $\left(\mathrm{F}_{(1)}=9.849 ; \mathrm{p}=.002\right)$.

Las comparaciones por pares (Tabla 7) muestran que en ambos grupos existen diferencias estadísticamente significativas entre el test y el retest y entre el test y el seguimiento en el tiempo de juego entre semana. Pero la diferencia entre el retest y el seguimiento únicamente es significativa en el grupo con técnicas de control de la impulsividad adicionales. La magnitud del cambio entre el test y el seguimiento, es decir, la magnitud del cambio total, es superior en el grupo con técnicas adicionales.

Tiempo de juego en fin de semana

Se aplicó un ANCOVA de medidas repetidas ( $3 \times 2$ ) para las mediciones realizadas del tiempo de juego en fin de semana, con un factor entre-grupos (condición programa tradicional vs. programa con técnicas de autocontrol adicionales) y un factor momento (test-retest-seguimiento).

Los resultados (Tabla 8) muestran un descenso del tiempo de juego en fin de semana a lo largo del tiempo (test-retestseguimiento) para los dos grupos. Puede apreciarse que 
Tabla 6. Tiempo de juego entre semana. Resultados del ANCOVA MR

\begin{tabular}{|c|c|c|c|c|c|c|c|c|c|c|c|}
\hline \multicolumn{4}{|c|}{ Momento } & \multicolumn{4}{|c|}{$\begin{array}{l}\text { Interacción } \\
\text { Momento x Grupo }\end{array}$} & \multicolumn{3}{|l|}{ Grupo } & \multirow[b]{2}{*}{ 1-b } \\
\hline $\mathrm{F}$ & $\mathbf{P}$ & $\mathbf{h}^{2}$ & $1-b$ & $\mathbf{F}$ & $\mathbf{p}$ & $\mathbf{h}^{2}$ & 1-b & $\mathbf{F}$ & p & $\mathbf{h}^{2}$ & \\
\hline 6.415 & .002 & .008 & 0.903 & 1.440 & .237 & .002 & .309 & 9.849 & .002 & .013 & .880 \\
\hline
\end{tabular}

Tabla 7. Comparaciones por pares para la interacción Tipo de Programa (Grupo) x Momento (Variable: tiempo de juego entre semana)

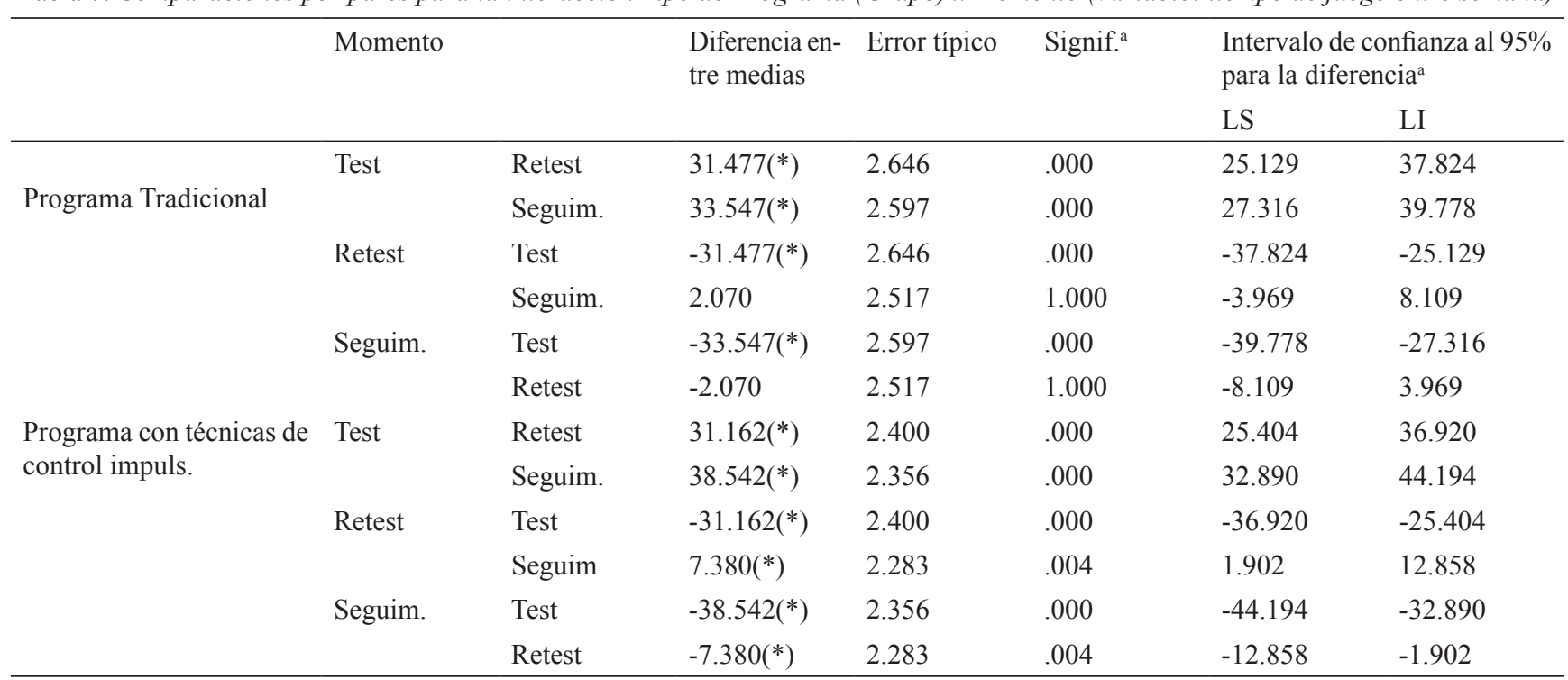

Nota. Basadas en las medias marginales estimadas.

* La diferencia de las medias es significativa al nivel .05.

a Ajuste para comparaciones múltiples: Bonferroni.

Tabla 8. Tiempo de juego en fin de semana. Medias y desviaciones típicas

\begin{tabular}{lccc}
\hline & Test & Retest & Seguimiento \\
\hline Programa tradicional & $98.77(62.89)$ & $70.09(68.62)$ & $63.68(69.09)$ \\
Programa con técnicas adicionales & $94.19(62.48)$ & $57.25(61.89)$ & $48.22(58.96)$ \\
\hline
\end{tabular}

el cambio es mayor en el grupo que realiza el programa con técnicas de control de la impulsividad adicionales, así mientras que en el test no existen diferencias entre grupos en esta variable, en el retest $(t=3,565 ; \mathrm{p}=0,000 ; \mathrm{d}=0,19)$ y en el seguimiento $(t=3,876 ; \mathrm{p}=0,000 ; \mathrm{d}=0,24)$ las diferencias son estadísticamente significativas.

El efecto del factor tiempo o momento de medida no resultó estadísticamente significativo. Se encuentra un efecto significativo del factor grupo $\left(\mathrm{F}_{(1)=} 8,614 ; \mathrm{p}=0,003\right)$ y de la interacción Tiempo x Momento $\left(\mathrm{F}_{(1,994)}=4,694 ; \mathrm{p}=0,009\right)$ (Tabla 9). Para examinar dicha interacción, se analizaron los efectos simples usando el ajuste de los niveles críticos mediante la corrección de Bonferroni para controlar la tasa de error Tipo I (Maxwell y Delaney, 1990).

Los resultados (Tabla 10) indican que en el grupo con técnicas de control de la impulsividad se producen diferencias en el tiempo de juego en fin de semana entre todos los momentos comparados (test, retest y seguimiento), mientras que en el caso del grupo al que se aplica el programa tradicional, existen diferencias estadísticamente significativas entre el test y el retest, y entre el test y el seguimiento, pero no entre el retest y el seguimiento. La magnitud del cambio, tanto entre el test-retest como entre el retest-seguimiento y entre el test-seguimiento, es mayor en el grupo con técnicas de control de la impulsividad (Figura 3 del Anexo I).

Diferencias en el Grado de Dependencia Percibida

Puesto que se hallaron diferencias iniciales en el grado de dependencia percibida entre los dos grupos, para poder corregir dichas diferencias y hacer los grupos comparables, se realizó un ajuste mediante el procedimiento de regresión. 
Tabla 9. Tiempo de juego en fin de semana. Resultados del ANCOVA MR

\begin{tabular}{|c|c|c|c|c|c|c|c|c|c|c|c|}
\hline \multicolumn{4}{|c|}{ Tiempo } & \multicolumn{4}{|c|}{$\begin{array}{l}\text { Interacción } \\
\text { Tiempo x Grupo }\end{array}$} & \multicolumn{4}{|l|}{ Grupo } \\
\hline $\mathrm{F}$ & $\mathbf{p}$ & $\mathbf{h}^{2}$ & 1-b & F & $\mathbf{p}$ & $\mathbf{h}^{2}$ & 1-b & F & $\mathbf{p}$ & $\mathbf{h}^{2}$ & 1-b \\
\hline 0.731 & .481 & .001 & 0.174 & 4.694 & $\begin{array}{l}.009 \\
\end{array}$ & .006 & .787 & 8.614 & .003 & .011 & .834 \\
\hline
\end{tabular}

Tabla 10. Comparaciones por pares para la interacción Tipo de Programa (Grupo) x Momento (Variable: tiempo de juego en fin de semana)

\begin{tabular}{|c|c|c|c|c|c|c|c|}
\hline & \multirow[t]{2}{*}{ Momento } & & \multirow[t]{2}{*}{$\begin{array}{l}\text { Diferencia en- } \\
\text { tre medias }\end{array}$} & \multirow[t]{2}{*}{ Error típico } & \multirow[t]{2}{*}{ Signif. ${ }^{a}$} & \multicolumn{2}{|c|}{$\begin{array}{l}\text { Intervalo de confianza al } 95 \% \\
\text { para la diferencia }{ }^{\mathrm{a}}\end{array}$} \\
\hline & & & & & & LS & LI \\
\hline \multirow{3}{*}{ Programa Tradicional } & Test & Retest & $27.995(*)$ & 3.193 & .000 & 20.333 & 35.657 \\
\hline & & Seguim. & $33.793(*)$ & 3.349 & .000 & 25.756 & 41.830 \\
\hline & Retest & Test & $-27.995(*)$ & 3.193 & .000 & -35.657 & -20.333 \\
\hline \multirow{9}{*}{$\begin{array}{l}\text { Programa con técnicas } \\
\text { adicionales de control } \\
\text { impulsividad }\end{array}$} & & Seguim. & 5.798 & 3.313 & .241 & -2.150 & 13.747 \\
\hline & Seguim. & Test & $-33.793(*)$ & 3.349 & .000 & -41.830 & -25.756 \\
\hline & & Retest & -5.798 & 3.313 & .241 & -13.747 & 2.150 \\
\hline & Test & Retest & $37.508(*)$ & 2.896 & .000 & 30.560 & 44.456 \\
\hline & & Seguim. & $47.043(*)$ & 3.037 & .000 & 39.755 & 54.331 \\
\hline & Retest & Test & $-37.508(*)$ & 2.896 & .000 & -44.456 & -30.560 \\
\hline & & Seguim & $9.535\left(^{*}\right)$ & 3.004 & .005 & 2.327 & 16.743 \\
\hline & Seguim. & Test & $-47.043\left(^{*}\right)$ & 3.037 & .000 & -54.331 & -39.755 \\
\hline & & Retest & $-9.535(*)$ & 3.004 & .005 & -16.743 & -2.327 \\
\hline
\end{tabular}

Nota. Basadas en las medias marginales estimadas.

* La diferencia de las medias es significativa al nivel .05.

a Ajuste para comparaciones múltiples: Bonferroni.

Para ello se realizó una regresión del pretest sobre el postest (retest y seguimiento) para cada grupo, de tal forma que se descontó el efecto del test sobre el retest y el seguimiento para el grado de dependencia percibida - para cada uno de los grupos-. Una vez se obtuvieron las medidas ajustadas, se procedió a la realización del ANCOVA de medidas repetidas. Se trata de un procedimiento de ajuste alternativo cuando las pendientes de regresión no son equivalentes en los diferentes grupos que se comparan y no es adecuado el uso del análisis de covarianza.

En las Tablas 11 y 12 puede observarse que el grado de dependencia percibida va descendiendo a lo largo de los distintos momentos de medida para ambos grupos. En el caso del grupo "tradicional", el cambio que se produce en dicha variable entre el test y el retest es estadísticamente significativo $\left(\mathrm{t}_{(398)}=6.819 ; \mathrm{p}=.000 ; \mathrm{d}=0.29\right)$ mientras que entre el retest y el seguimiento no lo es $\left(\mathrm{t}_{(347)}=1.408 ; \mathrm{p}=.160\right)$. Respecto al grupo con técnicas de control de la impulsividad, el cambio en el grado de dependencia percibida se produce de forma estadísticamente significativa tanto entre el test-retest $\left(\mathrm{t}_{(460)}=7.606 ; \mathrm{p}=.000 ; \mathrm{d}=0.27\right)$ como entre el retest-seguimiento $\left(\mathrm{t}_{(410)}=5.761 ; \mathrm{p}=.000 ; \mathrm{d}=0.21\right)$.

Los resultados del ANCOVA de medidas repetidas (Tabla 14) indican que efecto del factor tiempo o momento de medida resultó estadísticamente significativo $\left(\mathrm{F}_{(1,640)=} 48.458\right.$; $\mathrm{p}=.000$ ), también se encuentra un efecto significativo del factor grupo $\left(\mathrm{F}_{(1)=} 16.782 ; \mathrm{p}=.000\right)$ y de la interacción Tiempo $\mathrm{x}$ Momento $\left(\mathrm{F}_{(1,640)=} 3.483 ; \mathrm{p}=.040\right)$. Para examinar dicha interacción, se analizaron los efectos simples -es decir, se compararon entre sí los niveles del factor momento dentro de cada nivel del factor grupo-, usando el ajuste de los niveles críticos mediante la corrección de Bonferroni para controlar la tasa de error Tipo I (Maxwell y Delaney, 1990).

Los resultados (Tabla 15) indican que en los dos grupos se producen diferencias en el grado de dependencia percibida entre todos los momentos comparados (test, retest y seguimiento). En el grupo con técnicas de control de la impulsividad adicionales, la magnitud de dicho cambio es superior respecto al grupo que recibe el programa de prevención tradicional, tanto en el cambio total (test-seguimiento), como entre el retest y el seguimiento. 
Tabla 11. Evolución del grado de dependencia percibida en el grupo SIN

\begin{tabular}{llllll}
\hline & Test & Retest & Seguimiento & Test-Retest & Retest-Seguimiento \\
\hline Dependencia percibida & $37.01(27.73)$ & $29.11(26.66)$ & $27.57(25.93)$ & $t=6.819 \cdot p=.000$ & $t=1.408 ; p=.160$ \\
\hline
\end{tabular}

Tabla 12. Evolución del grado de dependencia percibida en el grupo CON

\begin{tabular}{llllll}
\hline & Test & Retest & Seguimiento & Test-Retest & Retest-Seguimiento \\
\hline Dependencia percibida & $32.75(27.34)$ & $25.82(24.53)$ & $20.99(23.03)$ & $t=7.606 ; p=.000$ & $t=5.761 ; p=.000$ \\
\hline
\end{tabular}

Tabla 13. Dependencia percibida. Medias y desviaciones típicas

\begin{tabular}{llll}
\hline & Test & Retest & Seguimiento \\
\hline Programa tradicional & $37.53(27.41)$ & $29.47(16.83)$ & $27.59(14.86)$ \\
Programa con técnicas adicionales & $33.28(28.25)$ & $26.16(18.25)$ & $22.05(16.01)$ \\
\hline
\end{tabular}

Tabla 14. Dependencia Percibida. Resultados del ANCOVA MR

\begin{tabular}{|c|c|c|c|c|c|c|c|c|c|c|c|}
\hline \multicolumn{4}{|l|}{ Tiempo } & \multicolumn{4}{|c|}{$\begin{array}{l}\text { Interacción } \\
\text { Tiempo x Grupo }\end{array}$} & \multicolumn{4}{|l|}{ Grupo } \\
\hline $\mathrm{F}$ & $\mathbf{p}$ & $\mathbf{h}^{2}$ & 1-b & $\mathbf{F}$ & $\mathbf{p}$ & $\mathbf{h}^{2}$ & 1-b & $\mathbf{F}$ & $\mathbf{p}$ & $\mathbf{h}^{2}$ & 1-b \\
\hline 48.458 & .000 & .048 & 1.000 & 3.483 & .040 & .004 & .592 & 16.782 & .000 & .017 & .984 \\
\hline
\end{tabular}

Tabla 15. Comparaciones por pares para la interacción Tipo de Programa (Grupo) x Momento (Variable: dependencia percibida).

\begin{tabular}{|c|c|c|c|c|c|c|c|}
\hline & \multirow[t]{2}{*}{ Momento } & & \multirow[t]{2}{*}{$\begin{array}{l}\text { Diferencia en- } \\
\text { tre medias }\end{array}$} & \multirow[t]{2}{*}{ Error típico } & \multirow[t]{2}{*}{ Signif. $^{\text {a }}$} & \multicolumn{2}{|c|}{$\begin{array}{l}\text { Intervalo de confianza al 95\% } \\
\text { para la diferencia }{ }^{\mathrm{a}}\end{array}$} \\
\hline & & & & & & LS & LI \\
\hline \multirow{3}{*}{ Programa Tradicional } & \multirow[t]{2}{*}{ Test } & Retest & $8.288(*)$ & .652 & .000 & 6.725 & 9.851 \\
\hline & & Seguim. & $10.214(*)$ & .688 & .000 & 8.565 & 11.864 \\
\hline & \multirow[t]{2}{*}{ Retest } & Test & $-8.288(*)$ & .652 & .000 & -9.851 & -6.725 \\
\hline \multirow{9}{*}{$\begin{array}{l}\text { Programa con técnicas } \\
\text { adicionales de control } \\
\text { impulsividad }\end{array}$} & & Seguim. & $1.926(*)$ & .443 & .000 & .864 & 2.988 \\
\hline & \multirow[t]{2}{*}{ Seguim. } & Test & $-10.214(*)$ & .688 & .000 & -11.864 & -8.565 \\
\hline & & Retest & $-1.926(*)$ & .443 & .000 & -2.988 & -.864 \\
\hline & \multirow[t]{2}{*}{ Test } & Retest & $6.933(*)$ & .594 & .000 & 5.508 & 8.357 \\
\hline & & Seguim. & $10.996(*)$ & .627 & .000 & 9.493 & 12.500 \\
\hline & \multirow[t]{2}{*}{ Retest } & Test & $-6.933(*)$ & .594 & .000 & -8.357 & -5.508 \\
\hline & & Seguim & $4.064(*)$ & .404 & .000 & 3.096 & 5.032 \\
\hline & \multirow[t]{2}{*}{ Seguim. } & Test & $-10.996(*)$ & .627 & .000 & -12.500 & -9.493 \\
\hline & & Retest & $-4.064(*)$ & .404 & .000 & -5.032 & -3.096 \\
\hline
\end{tabular}

Nota. Basadas en las medias marginales estimadas.

* La diferencia de las medias es significativa al nivel .05.

a Ajuste para comparaciones múltiples: Bonferroni.

\section{Diferencias en el TDV}

Puesto que se hallaron diferencias iniciales en el TDV entre los dos grupos, se realizó un ajuste mediante regresión, siguiendo el mismo procedimiento que para la variable "dependencia percibida".
Los resultados del ANCOVA de medidas repetidas (Tabla 17) indican que efecto del factor tiempo o momento de medida resultó estadísticamente significativo $\left(\mathrm{F}_{(1,000)}=42.793\right.$; $\mathrm{p}=.000$ ), también se encuentra un efecto significativo del factor grupo $\left(\mathrm{F}_{(1)=} 13.067 ; \mathrm{p}=.000\right)$ y de la interacción Tiempo $\mathrm{x}$ Momento $\left(\mathrm{F}_{(1,000)=} 5.085 ; \mathrm{p}=.024\right)$. Para examinar dicha 
interacción, se analizaron los efectos simples -es decir, se compararon entre sí los niveles del factor momento dentro de cada nivel del factor grupo-, usando el ajuste de los niveles críticos mediante la corrección de Bonferroni para controlar la tasa de error Tipo I (Maxwell y Delaney, 1990).

Los resultados (Tabla 18) indican que en los dos grupos se producen diferencias en el TDV entre el test y el seguimiento, siendo mayor la magnitud del cambio en el grupo con técnicas de control de la impulsividad adicionales.

\section{Discusión}

Las principales conclusiones que pueden extraerse de los resultados obtenidos son las siguientes:

1. La evolución del grupo que permanece en lista de espera muestra que cuando no se realiza la intervención (el programa de prevención en cualquiera de sus modalidades), los resultados no varían, es decir, se mantiene la misma frecuencia y tiempo de uso de los videojuegos. El hecho de que en el grupo en lista de espera no haya cambios significativos quiere decir que el paso del tiempo no ha sido el responsable de los efectos obtenidos en los grupos en los que en ese mismo periodo de tiempo se aplican las dos modalidades del programa de prevención.
Así pues, se demuestra que los resultados obtenidos se deben a la aplicación de los programas de prevención.

2. Los resultados encontrados al analizar la homogeneidad inicial de los grupos indican que no había diferencias estadísticamente significativas en la mayoría de las variables estudiadas antes de la aplicación del programa, aunque las había en la edad, el grado de dependencia percibido y en la puntuación total del TDV. En estas dos últimas variables, las medias relacionadas con las medidas de dependencia de videojuegos eran ligeramente superiores en el grupo en el que se aplicó el programa de prevención en su forma tradicional. Se llevó a cabo el control de estas diferencias iniciales -análisis de covarianza y ajuste mediante regresión del pretest sobre el postest (retest y seguimiento) para cada grupo-, puesto que se pretendía someter a prueba la eficacia diferencial de cada modalidad del programa.

3. En cuanto a los resultados obtenidos una vez aplicado el programa de prevención, los ANCOVAS de medidas repetidas realizados -teniendo en cuenta los datos obtenidos antes y después de la aplicación de las sesiones, así como el seguimiento- ponen de manifiesto que en los dos grupos se produce una disminución de las puntuaciones a lo largo de los distintos momentos de medida. Estos resultados ponen de manifiesto que

Tabla 16. TDV. Medias y desviaciones típicas

\begin{tabular}{lll}
\hline & Test & Seguimiento \\
\hline Programa tradicional & $28.88(21.50)$ & $21.17(13.27)$ \\
Programa con técnicas adicionales & $25.92(19.16)$ & $16.72(10.94)$ \\
\hline
\end{tabular}

Tabla 17. TDV. Resultados del ANCOVA MR

\begin{tabular}{llllllllllll}
\hline Tiempo & \multicolumn{1}{c}{ Interacción } \\
& \multicolumn{1}{c}{ Tiempo x Grupo } & \multicolumn{3}{c}{ Grupo } \\
$\mathrm{F}$ & $\mathrm{p}$ & $\mathrm{h}^{2}$ & $1-\mathrm{b}$ & $\mathrm{F}$ & $\mathrm{P}$ & $\mathrm{h}^{2}$ & $1-\mathrm{b}$ & $\mathrm{F}$ & $\mathrm{p}$ & $\mathrm{h}^{2}$ & $1-\mathrm{b}$ \\
\hline 42.793 & .000 & .048 & 1.000 & 5.085 & .024 & .006 & .615 & 13.067 & .000 & .015 & .951 \\
\hline
\end{tabular}

Tabla 18. Comparaciones por pares para la interacción Tipo de Programa (Grupo) x Momento (Variable: TDV).

\begin{tabular}{|c|c|c|c|c|c|c|c|}
\hline & \multirow[t]{2}{*}{ Momento } & & \multirow[t]{2}{*}{$\begin{array}{l}\text { Diferencia en- } \\
\text { tre medias }\end{array}$} & \multirow[t]{2}{*}{ Error típico } & \multirow[t]{2}{*}{ Signif.a } & \multicolumn{2}{|c|}{$\begin{array}{l}\text { Intervalo de confianza al 95\% } \\
\text { para la diferencia }\end{array}$} \\
\hline & & & & & & LS & LI \\
\hline Programa Tradicional & Test & Seguim. & $7.821(*)$ & .429 & .000 & 6.980 & 8.663 \\
\hline $\begin{array}{l}\text { Programa con técnicas } \\
\text { adicionales... }\end{array}$ & Test & Seguim. & $9.113(*)$ & .374 & .000 & 8.379 & 9.847 \\
\hline
\end{tabular}

Nota. Basadas en las medias marginales estimadas.

* La diferencia de las medias es significativa al nivel .05.

a Ajuste para comparaciones múltiples: Bonferroni. 
tras la aplicación del programa (en cualquiera de sus modalidades) los alumnos muestran un descenso del patrón de uso de los videojuegos así como menores puntuaciones en la dependencia de los mismos, y también queda patente la importancia de la prevención de las adicciones tecnológicas y la eficacia del módulo de videojuegos del programa PrevTec 3.1 (Marco et al., 2010, 2012).

4. Los resultados se mantienen en el seguimiento, que se realizó entre dos y cinco meses tras la finalización de la última sesión del programa de prevención. En el grupo que recibió el programa con técnicas de control de la impulsividad adicionales, las puntuaciones no solamente se mantuvieron en el seguimiento, sino que continuaron disminuyendo entre el retest y el seguimiento. Además, la magnitud del cambio que se produce en el grupo que recibe el programa con técnicas de control de la impulsividad es mayor que en el grupo que recibe el programa tradicional. Se puede afirmar, por tanto, que los dos programas son eficaces y que la condición que incluye técnicas de control de la impulsividad es superior.

5. Puesto que la única diferencia entre las dos versiones del módulo de videojuegos es el entrenamiento o no en técnicas específicas de control de la impulsividad, este resultado refleja dos aspectos significativos. Por un lado, la relevancia que tiene esta variable (la impulsividad) en la prevención y tratamiento de la adicción a videojuegos y de las adicciones tecnológicas; y por otro, la importancia de realizar un entrenamiento en técnicas conductuales, además de ofrecer información y realizar sensibilización en los programas de prevención. Probablemente el entrenamiento en conductas alternativas y de autocontrol del uso de los videojuegos sea un aspecto muy relevante, quizá porque para modificar y adquirir nuevos hábitos es necesario ofrecer conductas alternativas, y una vez logramos que los alumnos adquieran dichas conductas, pueden mantenerlas en el tiempo y así realizar un uso más adaptativo de estas herramientas.

\section{Referencias}

ADESE (Asociación Española de Distribuidores y Editores de Software de Entretenimiento) (2011). El videojugador español: perfil, hábitos e inquietudes de nuestros gamers. Recuperado desde http://www.adese. es/. Asociación Española de Distribuidores y Editores de Software de Entretenimiento.

Allison, S. E., von Wahlde, L., Shockley, T. , y Gabbard, G. O. (2006). The development of the self in the era of the Internet and role-playing fantasy games. American Journal of Psychiatry, 163, 381- 385.
Arnett, J. J. (1999). Adolescent Storm and Stress, Reconsidered. American Psychologist, 54, 317-326.

Bainbridge, W. S. (2007). The scientific research potential of virtual worlds. Science, 317, 472-476.

Batthyány, D., Müller, K. W., Benker, F., y Wölfling, K. (2009). Computer game playing: clinical characteristics of dependence and abuse among adolescents. Wiener Klinsche Wochenschrift, 121, 502-509.

Castellana, M., Sánchez-Carbonell, X., Beranuy, M., y Graner, C. (2006). La relació de l' adolescent amb les TIC: Un tema de rellevància social. Full Informatiu del Col-legi Oficial de Psicòlegs de Catalunya, 192, $22-23$

Castronova, E. (2005) Synthetic worlds: the business and culture of online games. Chicago: University of Chicago Press.

Chappell, D., Eatough, V. E., Davies, M. N. O., y Griffiths, M. D. (2006). EverQuest-It's just a computer game right? An interpretative phenomenological analysis of online game addiction. International Journal of Mental Health and Addiction, 4, 205-216. doi:10.1007/ s11469-006-9028-6.

Chiu, S., Lee, J., y Huang, D. (2004). Video Game Addiction in Children and Teenagers in Taiwan. CyberPsychology \& Behavior, 7, 571-581. doi:10.1089/cpb.2004.7.571

Chóliz, M. (2011). PrevTec 3.1: Programa de Prevención de Adicciones Tecnológicas. Valencia: FEPAD.

Chóliz, M., y Marco, C. (2011). Patrón de uso y dependencia de videojuegos en infancia y adolescencia. Anales de Psicología, 27, 418-426

Chóliz, M., Marco, C., y Chóliz, M. C. (2014). ADITEC. Madrid: TEA Ediciones.

Echeburua, E., y Requesens, A. (2012). Adicción a las redes sociales y a las nuevas tecnologías en jóvenes y adolescentes. Guía para educadores. Madrid: Piramide.

Egli, E. A., y Meyers, L. S. (1984). The role of videogame playing in adolescent life: is there reason to be concerned? Bulletin o the Psychonomic Society, 22, 309-312

Elliot, L., Golub, A., Ream, G., y Dunlap, E. (2012). Video Game Genre as a Predictor of Problem Use. Cyberpsycholog, Beahovior and Social Networking, 15, 155-161

Gentile, D. A. (2009). Pathological video-game use among youth ages 8 to 18: A national study. Psychological Science, 20, 594-602. doi: 10.1111/j.1467-9280.2009.02340.x

Griffiths, M. D. (1995). Technological addictions. Clinical Psychology Forum, 76, 14-19

Griffiths, M. D. (2000). Does Internet and computer "addiction" exists? Some case study evidence. CyberPsychology and Behavior, 3, 211-218

Griffiths, M. D. (2008). Digital impact, crossover technologies and gambling practices. Casino and Gaming International, 4, 37-42.

Griffiths, M. D., Davies, M. N., y Chappell, D. (2004). Online computer gaming: a comparison of adolescent and adult gamers. Journal of adolescence, 27, 87-96.

Griffiths, M. D., y Hunt, N. (1998). Dependence on computer games by adolescents. Psychological Reports, 82, 475-480.

Grüsser, S. M., Thalemann, R., y Griffiths, M. D. (2007). Excessive Computer Game Playing: Evidence for Addiction and Aggression? CyberPsychology \& Behavior, 10, 290-292.

Hart, G., Johnson, B., Stamm, B., Angers, N., Robinson, A., Lally, T., y Fagley, W. H. (2009). Effects of video games on adolescents and adults. CyberPsychology \& Behavior, 12, 63-65.

Jiménez-Albiar, M. I., Piqueras, J. A., Mateu-Martínez, O., Carballo, J. L., Orgilés, M., y Espada, J. P. (2012). Diferencias de sexo, características de personalidad y afrontamiento en el uso de Internet, el móvil y los videojuegos en la adolescencia. Salud y Drogas, 12, 61-82.

Keepers, G. A. (1990). Pathological preoccupation with video games. Journal of the American Academy of Child and Adolescent Psychiatry, 29, 49-50.

King, D. L., y Delfabbro, P. H. (2009). Understanding and assisting excessive players of video games: Acommunity psychology perspective. Australian Community Psychologist, 21, 62-74. 
Lee, M., Ko, Y., Song, H., Kwon, K., Lee, H., Nam, M., y Jung, I. (2007). Characteristics of Internet use in relation to game genre in Korean adolescents. Cyberpsychology \& Behavior, 10, 278-285

Lemmens J. S., Valkenburg P. M., y Peter J. (2009). Development and validation of a game addiction scale for adolescents. Media Psychology, 12, 77-95.

Lemmens, J. S. (2006). Gameverslaving. Probleemgebruik herkennen, begrijpen en overwinnen. Amsterdam: Uitgeverij SWP.

Machargo, J., Luján, I., León, M. E., López, P., y Martín, M. A. (2003). Videojuegos por los adolescentes. Anuario de Filosofia, Psicologia y Sociología, 6, 159-172.

Marco, C., Chóliz, M., y Martínez, E. (2012, septiembre). Técnicas de control de la impulsividad en la prevención de la adicción a videojuegos. Comunicación presentada en el IX Congreso Internacional de la Sociedad Española para el Estudio de la Ansiedad y el Estrés. Valencia, España.

Marco, C., Villanueva, V., y Chóliz, M. (2010). Prevención de la adicción a videojuegos en la adolescencia: resultados del programa PrevTec 3.1. En L. Ferrer y L. Cabello (Coords.) Avances en Tratamientos Psicológicos (pág. 125). Granada. Asociación Española de Psicología Conductual.

Maxwell, S. E., y Delaney, H. D. (1990). Designing experiments and analyzing data: A model comparison perspective. Belmont, CA: Wadsworth.

Meerkerk, G. J., Van Den Eijnden, R., Vermulst, A. A., y Garretsen, H. F. L. (2009). The Compulsive Internet Use Scale (CIUS): some psychometric properties. Cyberpsychology \& Behavior, 12, 1-6.

Patim (2009). Memoria Anual de las Actividades del Centro Guillem Despuig de Adicciones No Tóxicas. Documento interno no publicado.

Peters, C. S., y Malesky, A. (2008). Problematic usage among highlyengaged players of massively multiplayer online role playing games. Cyberpsychology \& Behavior, 11, 481-484.

Peukert, P., Sieslack, S., Barth, G., y Batra, A. (2010). Internet- and computer game addiction: phenomenology, comorbidity, etiology, diagnostics and therapeutic implications for the addictives and their relatives. Psychiatrische Praxis, 3, 219-224. doi:10.1055/s-0030-1248442

Phillips, C. A., Rolls, S., Rouse, A., y Griffiths, M. D. (1995). Home video game playing in schoolchil-dren: A study of incidence and patterns of play. Journal of Adolescence, 18, 687-691.

Rehbein, F., Kleimann, M., y Mößle, T. (2010). Prevalence and risk factors of video game dependency in adolescence: Results of a German nationwide survey. CyberPsychology, Behavior and Social Networking, $13,269-277$.

Rubia, F. (2004). La corteza prefrontal, órgano de la civilización. Revista de Occidente, 272, 88-97.

Saldaña, D. (2001). Nuevas tecnologías: nuevos instrumentos y nuevos espacios para la psicología. Apuntes de Psicología, 19, 5-10.

Skoric, M. M., Teo, L. L. C., y Neo, R. L. (2009). Children and video games: addiction, engagement, and scholastic achievement. Cyberpsychology \& Behavior, 12, 567-572

Smyth, J. (2007). Beyond self-selection in video game play: an experimental examination of the consequences of massively multiplayer online role-playing game play. CyberPsychology \& Behavior, 10, 717-721.

Spear, L. P. (2000a). The adolescent brain and age-related behavioral manifestations. Neuroscience and Biobehavioral Reviews, 24, 417-463.

Spear, L. P. (2007b). The psychobiology of adolescence. En K. Kline (Ed.), Authoritative Communities: The Scientific Case for Nurturing Children in Body, Mind, and Spirit. Nueva York: Springer Publishing.

Steinberg, L. (2005). Cognitive and affective development in adolescence. Trends in Cognitive Sciences, 9, 69-74.

Tanner, L. (2007). AMA considers classifying video game addiction as a mental illness. Associated Press. Recuperado desde http://www. foxnews.com/story/ 0,2933,285558,00.html

Tejeiro, R. A., y Bersabé, R. M. (2002). Measuring problem video game playing in adolescents. Addiction, 97, 1601-1606

Van Cleave, R. (2010). Unplugged: my journey into the dark world of video game addiction. Deerfield Beach, FL: Health Communications, Inc.

Van den Eijnden, R. J. J. M., Van Rooij, A. J., y Meerkerk, G. J. (2007). Excessief en Compulsief Internetgebruik. Een Kwalitatieve Analyse
[Excessive and Compulsive Internet use. A Qualitative Analysis] (Factsheet). Rotterdam: IVO. Recuperado desde http://www.nji.nl/ smartsite.dws?id=133840.

Van Rooij, A. J. (2011). Online Video Game Addiction. Exploring a new phenomenon (Tesis Doctoral). Erasmus University Rotterdam, The Netherlands. Recuerado desde http://repub.eur.nl/res/pub/23381/.

Van Rooij, A. J., y Van den Eijnden, R. J. J. M. (2007). Monitor Internet en Jongeren 2006 en 2007. Ontwikkelingen in Internetgebruik en de rol van opvoeding [Monitor Internet and Youth 2006 and 2007. Developments in Internet Use and the Role of Parenting] (IVO Reeks No. 54). Rotterdam: IVO. Recuperado desde http://www.ivo.nl/UserFiles/File/ Publicaties/200710\%20IVO\%20Reeks\%2054\%20\%20Monitor\%20 Internet $\% 20$ en $\% 20 J$ ongeren $\% 202006 \% 20$ en $\% 202007 . p d f$

Van Rooij, A. J., Schoenmakers, T. M., van de Eijnden, R., y van de Mheen, D. (2010). Compulsive Internet use: The role of online gaming and other Internet applications. Journal of Adolescent Health, 47, 51-57.

Villanueva, V., Marco, C., y Chóliz, M. (2010a). Prevención de la adicción al móvil en la adolescencia: resultados del programa PrevTec 3.1. En L. Ferrer y L. Cabello (Coords.) Avances en Tratamientos Psicológicos (pág. 124). Granada. Asociación Española de Psicología Conductual.

Villanueva, V., Marco, C., y Chóliz, M. (2010b). Prevención de la adicción a Internet en la adolescencia: resultados del programa PrevTec 3.1. Comunicación presentada en el VII Congreso Iberoamericano de Psicología. Oviedo.

Villanueva, V., Marco, C., y Chóliz, M. (2012). Programa de prevención del abuso y la dependencia del teléfono móvil en población adolescente. En I. Montero, MJ. De Dios, B. Sierra y J.A. Huertas (Eds.). La investigación en Motivación y Emoción: Contribuciones de jóvenes investigadores en formación (pp. 217-239). Publicación electrónica: AME.

Weinberger, D. R., Elvevag, B., y Giedd, J. N. (2005). The adolescent brain: A work in progress. Washington, DC: National Campaign to Prevent Teen Pregnancy.

Yee, N. (2006). The demographics, motivations, and derived experiences of users of massively-multiuser online graphical environments. Presence: Teleoperators and Virtual Environments, 15, 309-329. 
